\title{
Dyssynergia Cerebellaris Myoclonica
}

National Institute of Neurological Disorders and Stroke (NINDS)

\section{Source}

National Institute of Neurological Disorders and Stroke (NINDS). Dyssynergia

Cerebellaris Myoclonica Information Page.

Dyssynergia Cerebellaris Myoclonica refers to a collection of rare, degenerative,

neurological disorders characterized by epilepsy, cognitive impairment, myoclonus, and

progressive ataxia. Symptoms include seizures, tremor, and reduced muscle coordination. Onset of the disorder generally occurs in early adulthood. Tremor may begin in one extremity and later spread to involve the entire voluntary muscular system. Arms are usually more affected than legs. Some of the cases are due to mitochondrial abnormalities. 